\title{
The Toronto Observational Study of Natalizumab in Multiple Sclerosis
}

\author{
Kristen M. Krysko, Paul W. O’Connor
}

\begin{abstract}
Background: Natalizumab is indicated for the treatment of relapsing multiple sclerosis (MS) with insufficient response to first-line disease-modifying therapy (DMT). We studied the efficacy of natalizumab for treatment of MS in a single centre observational design. Methods: A retrospective observational study of 146 patients [66\% female; mean age 37.4; 72\% relapsing remitting MS (RRMS), 28\% secondary progressive MS (SPMS)] referred for natalizumab treatment at St. Michael's Hospital MS Clinic between 2007 and August 2009. Data included demographic, clinical (Expanded Disability Status Scale (EDSS) and annualized relapse rate (ARR)) and patient self-report measures. Results: The mean duration of treatment was 20 months in those treated with natalizumab and $97 \%$ had received prior DMTs. Eighty-three patients (57\%) received at least 12 months of natalizumab treatment. In those who received at least 12 months of treatment, baseline ARR and EDSS were 1.6 and 2.7 in RRMS patients versus 1.0 and 5.4 in SPMS with relapses. The ARR decreased with natalizumab treatment to 0.38 (76\% reduction, $\mathrm{p}<0.001)$ in RRMS versus 0.32 in SPMS patients ( $68 \%$ reduction, $\mathrm{p}=0.01$ ). There was a treatment associated $11 \%$ reduction in EDSS to 2.4 ( $\mathrm{p}=0.04)$ in RRMS, but no significant change in SPMS. Eighty-five percent of patients reported improved overall quality of life (QOL) and 62\% indicated improved energy. Conclusions: There was a major reduction in relapse rate, stabilization in EDSS and improvement in QOL and energy in some patients on natalizumab, all similar to treatment effects in the pivotal trial.
\end{abstract}

RÉSUMÉ: Le Toronto Observational Study of Natalizumab dans la sclérose en plaques. Contexte : Le natalizumab est indiqué dans le traitement de la sclérose en plaques (SP) rémittente qui ne répond pas suffisamment au traitement de fond (TF) de première ligne. Nous avons utilisé un plan d'étude d'observation dans un seul centre pour étudier l'efficacité du natalizumab pour traiter la SP. Méthode : Il s'agit d'une étude d'observation rétrospective portant sur 146 patients atteints de SP [72\% de SP rémittente (SPR) et 28\% de SP secondairement progressive (SPSP)], dont 66\% étaient des femmes et dont l'âge moyen était de 37,4 ans. Ils avaient été référés à la clinique de SP de St. Michael's Hospital pour traitement par le natalizumab entre 2007 et août 2009. Nous avons recueilli les données démographiques, le score à l'Expanded Disability Status Scale (EDSS) et le taux annualisé de rechute (TAR) et les mesures rapportées par les patients. Résultats : La durée moyenne de traitement était de 20 mois chez les patients traités par le natalizumab et $97 \%$ ont reçu d'autres TF. Quatre-vingt-trois patients (57\%) ont reçu le natalizumab pendant au moins 12 mois. Chez ces patients, le TAR et l'EDSS avant traitement étaient de 1,6 et 2,7 respectivement chez les patients atteints de SPR et de 1,0 et 5,4 chez ceux atteints de SPSP qui ont eu des récidives. Le TAR a diminué sous traitement par le natalizumab à 0,38 (diminution de $76 \%, \mathrm{p}<0,001$ ) chez les patients atteints de $\mathrm{SPR}$ et à 0,32 chez ceux atteints de SPSP (diminution de $68 \%, \mathrm{p}=0,01$ ). Nous avons observé une diminution de $11 \%$ du score à l'EDSS associée au traitement, soit 2,4 chez les patients atteints de SPR $(\mathrm{p}=0,04)$. Il n'y a eu aucun changement significatif du score à l'EDS chez ceux atteints de SPSP. Quatre-vingtcinq pour cent des patients ont noté une amélioration globale de leur qualité de vie $(\mathrm{QV})$ et $62 \%$ ont noté un niveau d'énergie plus élevé. Conclusions : Sous natalizumab, certains patients ont noté une diminution importante du taux de récidive, une stabilisation du score à l'EDSS et une amélioration de leur QV et de leur niveau d'énergie, ce qui est similaire aux effets observés dans l'étude pivot.

Can. J. Neurol. Sci. 2011; 38: 422-428

Relapsing-remitting multiple sclerosis (RRMS) is a demyelinating neurological disease characterized by the development of inflammatory lesions in the central nervous system. Current first line disease modifying therapies (DMT) include interferon beta-1a, interferon beta- $1 \mathrm{~b}$ and glatiramer acetate. These are only partially effective, with most patients experiencing breakthrough disease activity. ${ }^{1-4}$

Natalizumab is a humanized monoclonal antibody indicated as a second-line monotherapy for the treatment of relapsing multiple sclerosis (MS) with insufficient response to first-line disease-modifying therapy or aggressive MS. Natalizumab is an $\alpha_{4}$ integrin antagonist, which binds $\alpha_{4}$ integrin on leukocytes and inhibits their migration into the central nervous system. This reduces inflammation in the brain through targeting a key component in the pathogenesis of lesion formation in MS..$^{5-7}$
Pivotal phase III randomized controlled trials including AFFIRM (Natalizumab Safety and Efficacy in Relapsing Remitting Multiple Sclerosis study) and SENTINEL (Safety and Efficacy of Natalizumab in Combination with Interferon Beta-1a in Patients with Relapsing Remitting Multiple Sclerosis study) have provided evidence for the efficacy of natalizumab for use in relapsing remitting MS. In phase III AFFIRM, ${ }^{8}$ natalizumab reduced sustained disability progression by $42 \%$ over two years

\footnotetext{
From the Division of Neurology, St. Michael's Hospital, Toronto, Ontario, Canada. Received October 26, 2010. Final Revisions Submitted January 10, 2011. Correspondence to: Paul O'Connor, MS Clinic and MS Research, St. Michael's Hospital, 30 Bond St \#3007S, Toronto, Ontario, M5B 1W8, Canada.
} 
and reduced rate of relapse by $68 \%$ over one year. In phase III SENTINEL, ${ }^{9}$ combination of natalizumab + interferon- $\beta 1 \mathrm{a}$ reduced sustained disability progression by $24 \%$ over two years and reduced rate of relapse by $54 \%$ over one year compared to interferon- $\beta 1 \mathrm{a}$ alone. Additionally, AFFIRM and SENTINEL showed improvement in health-related physical and mental quality of life with natalizumab use as measured by the short form health survey. ${ }^{10}$

Several post-marketing observational studies have been conducted in Europe to investigate the safety and effectiveness of natalizumab as a second line monotherapy for MS in clinical practice. These studies have consistently found reduction in annualized relapse rate and stabilization of Expanded Disability Status Scale (EDSS) scores while on natalizumab, providing support for the effectiveness of natalizumab in clinical practice in Europe. ${ }^{11-17}$ The use of natalizumab in clinical practice has not yet been formally studied in Canada.

Observational studies have many limitations, including a lack of blinding and randomization, which can lead to bias. However, randomized control trials limit generalizability and it is important to also study the effectiveness of drug treatments in clinical practice. Thus, we aim to study the effectiveness and safety of natalizumab in clinical practice in Toronto and to compare findings with those of the pivotal trials.

\section{METHODS}

This study was a retrospective observational review of 146 patients referred for natalizumab treatment in clinical practice at the Multiple Sclerosis Clinic at St. Michael's Hospital (SMH) in Toronto. The study protocol was developed by the authors and was approved by the Research Ethics Board at SMH.

Inclusion criteria included patients of 18 years-of-age or older diagnosed with MS and prescribed natalizumab treatment at St. Michael's Hospital MS Clinic between 2007 and August 2009. For inclusion in the full analyses, patients were required to have received at least 12 months of natalizumab treatment by August 2010. Thus, patients who discontinued natalizumab prior to 12 months of treatment and those who had not yet been treated for 12 months at the time of data collection were excluded from analyses.

This was a retrospective observational review of patient charts. Patients treated with natalizumab at SMH were seen in clinic every six to eight months, during which relapses were reported and the EDSS score or neurologic examination were performed and documented in the medical chart. Chart review involved recording demographic data, MS history and prior use of DMTs. Patients were categorized based on clinical course of their MS, which included RRMS (experiencing relapses without evidence of progression), secondary progressive MS (SPMS) with relapses (evidence of progression, but at least one relapse in the two years before starting natalizumab), or SPMS (evidence of progression with no relapses in the two years prior to starting natalizumab). Neurologist-determined relapses recorded in the medical chart were used to extract the annualized relapse rate (ARR) in the one, two and three years prior to natalizumab treatment and the ARR during natalizumab treatment. Similarly, the EDSS score was determined at the visit prior to beginning natalizumab and after $12+/-2$ months of natalizumab treatment. The EDSS score after $24+/-2$ months of treatment was recorded when available. Expanded Disability Status Scale was extracted from patient charts when it was listed. In cases where EDSS was not listed explicitly, it was determined through the use of information regarding physical examination findings, ambulation distance and ambulatory assistance required, which were listed in patient charts.

Subjective effects of natalizumab were recorded based on patients' responses to an open-ended question ("How has your life changed since beginning Tysabri?") provided by the Tysabri Care Program ${ }^{\mathrm{TM}}$ and included in the medical chart. This qualitative data was analyzed for themes. For each theme that emerged, responses were categorized into improved, stable, worsened and mixed change (initial improvement after each infusion which declined with time to the next infusion, which has been referred to as a "wearing off effect"). This data was displayed in graphical format.

Primary outcome measures included the change in annualized relapse rate and the change in EDSS score with 12 and 24 months of natalizumab treatment in both patients with RRMS and SPMS with relapses who were treated for at least 12 months. Percentage of relapse-free patients and progression-free patients $(<1.0$ worsening in EDSS) after 12 months of natalizumab treatment were also determined. Self-report qualitative data was analyzed for themes that patients indicated changed with natalizumab use; this was a secondary outcome measure. Findings were compared with those from AFFIRM $^{8}$ and SENTINEL. ${ }^{9}$

IBM Statistical Package for the Social Sciences Statistics 19 was used for data analyses. Dependent t-tests were used to compare baseline ARR and EDSS with ARR and EDSS during natalizumab treatment in patients with RRMS and SPMS with relapses.

\section{Results \\ Patients}

One hundred and forty-six patients were referred for natalizumab treatment at the SMH MS Clinic between 2007 and August 2009. Patient demographics were similar to those who participated in $\mathrm{AFFIRM}^{8}$ and SENTINEL, ${ }^{9}$ with $66 \%$ female and a mean age of 37.4 years. However, our participants had a longer duration of disease (median eight years) compared to the pivotal trials. Our study included 105 (72\%) with RRMS, 32 (22\%) with secondary progressive MS with relapses, and 9 (6\%) with SPMS, while AFFIRM ${ }^{8}$ and SENTINEL ${ }^{9}$ only included RRMS (Table). There were no patients with primary progressive MS.

Patient ethnicity was only recorded in $36 \%$ of patient charts (missing from 64\%), while language fluency was not recorded in charts. Of the 52 patients whose chart included ethnicity, there were 32 European (62\%), 9 Asian (17\%), 6 African (12\%), 2 Caribbean (4\%), 2 Middle Eastern (4\%), and 1 Aboriginal (2\%).

Of the 146 patients referred for natalizumab, $83(57 \%)$ completed at least 12 months of treatment by August 2010, with 58 of these continuing treatment and 25 discontinued. Twentysix patients $(18 \%)$ were treated for less than 12 months, with 8 continuing treatment and 18 discontinued before 12 months of treatment. Thirty-seven patients $(26 \%)$ chose not to begin treatment despite being prescribed natalizumab. Of the patients who began treatment (109 patients), 66 patients continue 
Table: Baseline characteristics of natalizumab treated patients

\begin{tabular}{|c|c|c|c|c|}
\hline Characteristic & $\begin{array}{l}\text { SMH patients referred } \\
\text { for natalizumab }(n=146)\end{array}$ & $\begin{array}{l}\text { SMH patients } \\
\text { treated for at least } \\
12 \text { months }(n=83) \\
\end{array}$ & $\begin{array}{l}\text { AFFIRM } \\
\text { treatment group } \\
(\mathrm{n}=627)\end{array}$ & $\begin{array}{l}\text { SENTINEL } \\
\text { treatment group } \\
(\mathrm{n}=589)\end{array}$ \\
\hline $\begin{array}{c}\text { Gender, } \mathbf{n}(\%) \\
\text { Female }\end{array}$ & $97(66 \%)$ & $52(63 \%)$ & $449(72 \%)$ & $442(75 \%)$ \\
\hline $\begin{array}{l}\text { Age, years } \\
\text { Mean }+/- \text { SD }\end{array}$ & $37.42+/-9.90$ & $37.04+/-9.17$ & $35.6+/-8.5$ & $38.8+/-7.7$ \\
\hline $\begin{array}{l}\text { Clinical Course, n (\%) } \\
\text { RRMS } \\
\text { SP \& relapsing } \\
\text { SPMS } \\
\end{array}$ & $\begin{array}{l}105(72 \%) \\
32(22 \%) \\
9(6 \%) \\
\end{array}$ & $\begin{array}{l}57(69 \%) \\
21(25 \%) \\
5(6 \%) \\
\end{array}$ & $627(100 \%)$ & $589(100 \%)$ \\
\hline $\begin{array}{l}\text { Disease duration, years } \\
\text { Median (range) }\end{array}$ & $8(0-27)$ & $9(0-27)$ & $5(0-34)$ & $7(1-34)$ \\
\hline
\end{tabular}

Baseline characteristics of patients referred for natalizumab treatment at St. Michael's Hospital. St. Michael's Hospital patients who received at least 12 months of natalizumab (primary outcome measures were analyzed in this group), and AFFIRM $^{8}$ and SENTINEL $^{9}$ treatment groups

treatment (61\%) and $43(39 \%)$ have discontinued (Figure 1).

Among the 109 patients who began natalizumab treatment, the mean duration of treatment was $20+/-10$ months of treatment (missing data from one patient). Among the 83 patients with at least 12 months of treatment, the mean duration of treatment was $24+/-7$ months (missing data from one patient). Most patients (97\%) had used at least one prior DMT for MS, which include intramuscular interferon- $\beta$-1a (Avonex®), subcutaneous interferon- $\beta$ - 1 a $\quad$ (Rebif $\AA$ ), subcutaneous interferon- $\beta$ - 1 b (Betaseron $\AA)$, glatiramer acetate (Copaxone $\AA)$, cyclophosphamide, mitoxantrone, azathioprine (Imuran $\AA$ ), teriflunomide and fingolimod. Only $3 \%$ of patients were DMT naïve.

The majority of patients were referred for natalizumab due to failure of previous DMTs $(70 \%)$, with continued relapses despite treatment. Others were referred for natalizumab due to intolerance of DMTs (23\%), new onset very aggressive MS (3\%), or due to a strong patient desire for natalizumab treatment $(4 \%)$. Data was missing from one patient.

\section{Effect on annualized relapse rate (ARR)}

The 83 patients who completed at least 12 months of natalizumab treatment were analyzed for the effect of natalizumab on annualized relapse rate through dependent ttests. In RRMS patients $(n=57)$, the baseline ARR was 1.6 relapses per year in the one year prior to starting natalizumab. In this group, ARR decreased with natalizumab treatment to 0.38 relapses per year. This represents a $76 \%$ reduction in ARR with natalizumab treatment in RRMS $(\mathrm{t}(56)=7.027, \mathrm{p}<0.001)$. The baseline ARR in the two and three years prior to natalizumab were both 1.1 relapses per year, resulting in a $65 \%$ reduction in ARR with natalizumab treatment when compared to the ARR in the two and three years prior to treatment. Additionally, of those with RRMS treated for at least 12 months $(n=57), 38$ were relapse-free $(67 \%)$, while 19 patients experienced one or more relapse while on natalizumab. In AFFIRM, 77\% were relapsefree at 12 months and $67 \%$ were relapse-free at 24 months. ${ }^{8}$

In patients with SPMS with relapses $(n=21)$, the baseline ARR was 1.0 relapses per year in the one year prior to natalizumab treatment. Natalizumab treatment was associated with a $68 \%$ reduction in ARR to 0.32 relapses per year $(\mathrm{t}(20)=2.727, \mathrm{p}=0.013)$. The baseline ARR was also 1.0 in the two and three years prior to natalizumab treatment in this group. Additionally, of those with SPMS with relapses treated for at least 12 months $(n=21), 13$ were relapse-free $(62 \%)$, while 8

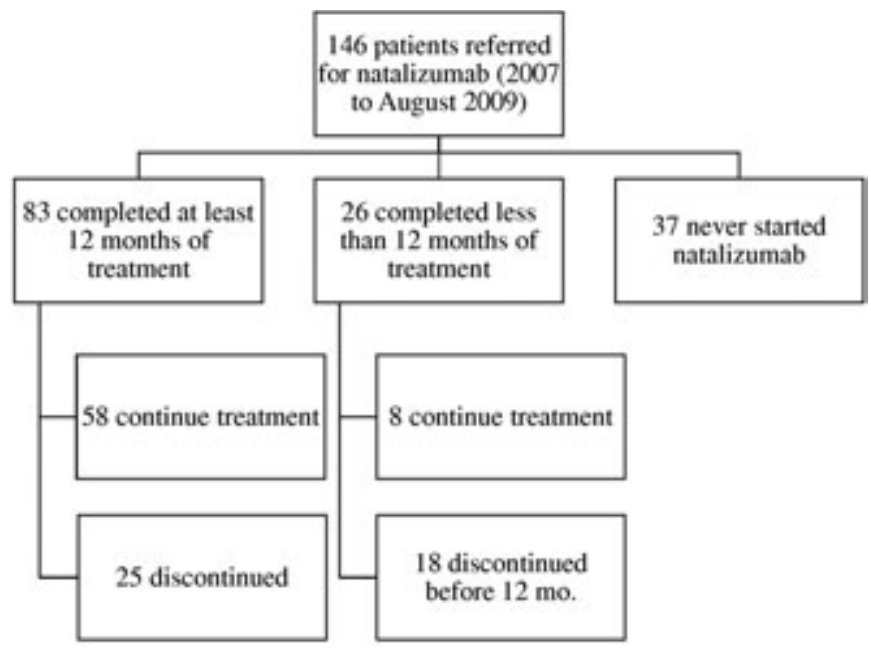

Figure 1: Patient flowchart showing status of natalizumab treatment as of August 2010. 


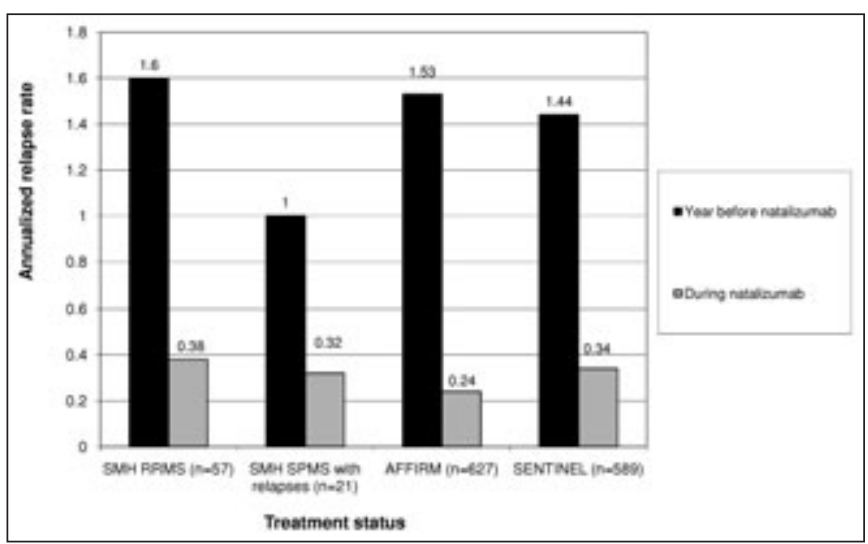

Figure 2: Annualized relapse rate change with natalizumab use in RRMS $S M H, S P M S$ SMH, AFFIRM ${ }^{8}$ and SENTINEL $L^{9}$ groups. There was a $76 \%$ reduction in ARR in RRMS $(p<0.001)$ and a $68 \%$ reduction in ARR in $S P M S$ with relapses $(p=0.01)$.

patients experienced one or more relapse while on natalizumab. The five patients with SPMS without relapses who completed 12 months of natalizumab were not included in analyses.

The effect on ARR was similar to findings in $\mathrm{AFFIRM}^{8}$ and SENTINEL $^{9}$ for those with RRMS. Those with SPMS with relapses similarly had a reduction in ARR with natalizumab treatment (Figure 2).

\section{Effect on disability (EDSS)}

Expanded Disability Status Scale scores were explicitly listed in $63 \%$ of cases, while $37 \%$ of EDSS scores were calculated based on physical examination findings, ambulation distance and ambulatory assistance listed in patient charts. The mean time between baseline EDSS determination and the most recent relapse was 10.8 months.

In patients with RRMS who completed at least 12 months of treatment $(n=57)$, the baseline EDSS was 2.7. Treatment with natalizumab for 12 months was associated with an $11 \%$ reduction in mean EDSS to $2.4(\mathrm{t}(55)=2.084, \mathrm{p}=0.042)$. For those who completed 24 months of treatment $(n=27)$, EDSS decreased to 2.0, but this was not significantly different from baseline $(\mathrm{t}(26)=1.744, \mathrm{p}=0.093)$. Additionally, of those with RRMS who completed at least 12 months of treatment, $49(86 \%)$ were progression-free (less than 1.0 worsening of EDSS over 12 months), while seven worsened in EDSS score by 1.0 or more (12\%). Thirty percent of patients improved in EDSS by at least 1.0 , while $56 \%$ were within one EDSS of baseline at 12 months. Twelve-month EDSS data was missing from one patient, while 24-month EDSS score was missing from 30 patients. The effect on EDSS was similar to findings in AFFIRM $^{8}$ and SENTINEL ${ }^{9}$, with stabilization of disability in RRMS (Figure 3).

In patients with SPMS with relapses who completed at least 12 months of natalizumab treatment $(n=21)$, the baseline EDSS was 5.36. Despite natalizumab treatment, EDSS score increased to 5.55 over 12 months of natalizumab treatment, but this increase was not significant $(\mathrm{t}(20)=0.969, \mathrm{~ns})$. In those who were treated for at least 24 months $(n=9)$, EDSS score increased to 5.72 over 24 months of treatment, which was also not significant

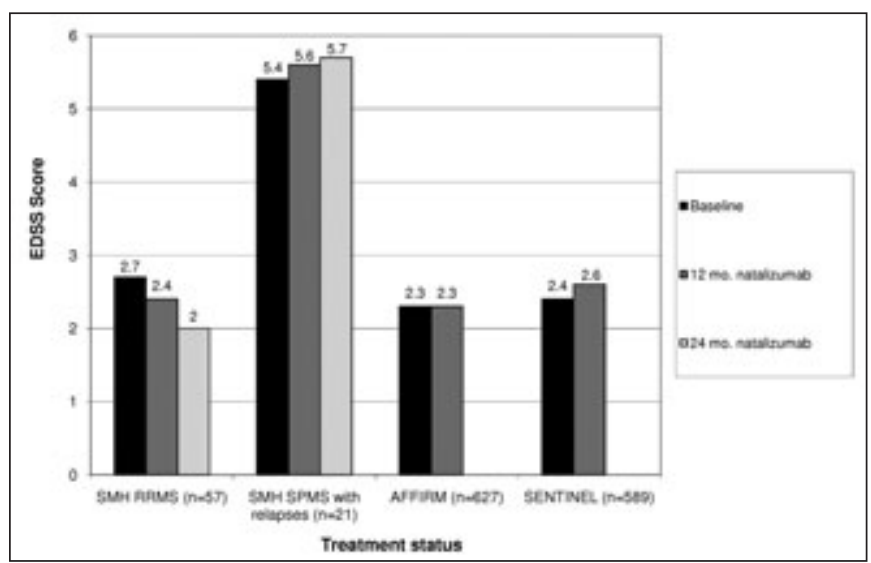

Figure 3: Expanded disability status scale change with 12 and 24 months of natalizumab use in RRMS SMH, SPMS SMH, AFFIRM ${ }^{8}$ and SENTINEL ${ }^{9}$ groups (Biogen Idec, data on file). There was an $11 \%$ reduction in EDSS with 12 months of natalizumab in RRMS ( $p=0.04)$, while there was no significant change in EDSS in SPMS with relapses.

$(\mathrm{t}(8)=2.052$, ns) (Figure 3). Additionally, of those with SPMS with relapses who completed at least 12 months of treatment, 17 (81\%) were progression-free (less than 1.0 worsening of EDSS over 12 months), while four worsened in EDSS score by 1.0 or more (19\%). Four percent of patients improved in EDSS by at least 1.0, while $62 \%$ were within one EDSS of baseline at 12 months. Twenty-four month EDSS data was missing from 12 patients in this group.

\section{Subjective effects of natalizumab}

Patients provided open-ended reports of the subjective effects of natalizumab in their charts through optional response to the question: "How has your life changed since beginning Tysabri?" There was a $68 \%$ response rate to this question. This data was analyzed qualitatively for themes. Themes that emerged during analyses included quality of life, energy, cognitive function, strength and balance. Forty-six patients noted an impact on quality of life (QOL), with 85\% noting improvement. Nine percent noted initial improvement in QOL after a natalizumab infusion, which decreased with time to the next infusion. Seven percent noted a decrease in QOL with treatment (missing data from 63 patients). Fifty-five patients noted an impact of natalizumab on energy, with $62 \%$ noting improved energy, $5 \%$ noting worsened energy and $15 \%$ noting initial improvement in energy after each infusion which declined until the next infusion (missing data from 54 patients). This was similar to findings in the pivotal trials ${ }^{10}$ which showed improved quality of life and vitality in patients treated with natalizumab.

Eighteen patients noticed an impact on cognitive function, with $61 \%$ noting improvement in memory/cognition and $17 \%$ noting worsening in cognitive function (missing data from 91 patients). Reports of impact on strength (missing data from 82 patients) and balance (missing data from 86 patients) were also made, but reports were quite varied (Figure 4). 


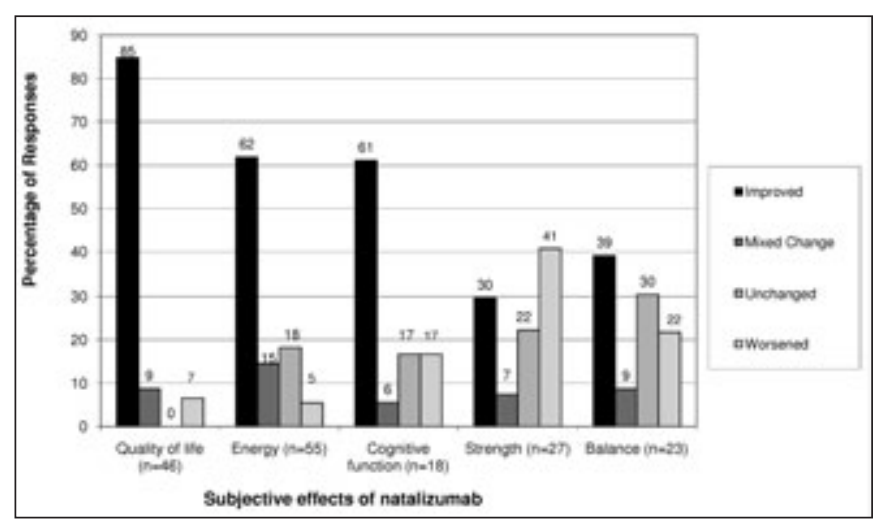

Figure 4: Percentage of SMH patients reporting various subjective effects of natalizumab use.

\section{Reasons for discontinuing}

As mentioned, 43 patients $(39 \%)$ discontinued treatment by August 2010. The mean duration of treatment before discontinuing was $15+/-11$ months. Reasons for discontinuing included an allergic reaction with hives in 11 patients $(26 \%)$, risks outweighing benefits of continued treatment in $11(26 \%)$, worsening disease requiring more aggressive treatment in 7 $(16 \%)$, lack of efficacy in $5(11 \%)$, non-allergic side effects in 4 (9\%), discontinued funding in $3(7 \%)$, desire for pregnancy in 1 $(2 \%)$ and 1 deceased $(2 \%)$.

Allergic reactions with hives occurred in 11 patients (10\% of treated patients) after a mean of $3+/-2$ infusions. Six reactions occurred after the second infusion, while one allergic reaction occurred after each of the first, third, fourth, fifth and tenth infusions. In nine patients these reactions were only cutaneous and non-severe and were treated in the infusion centre with histamine antagonists. However, two patients (after their second infusion) were sent to a local emergency department due to symptoms of anaphylaxis. One patient required epinephrine, and both required steroid treatment in addition to histamine antagonists. All patients had full recovery. Of the 11 patients with allergic reactions, two had positive anti-natalizumab antibodies (18\%). These were the only two patients who were positive for anti-natalizumab antibodies in our study (routinely tested at six months of treatment); thus, the overall rate of positive neutralizing antibodies was $1.8 \%$.

Eleven patients discontinued treatment after two years due to risks outweighing the benefits of continued treatment with prolonged duration of treatment as determined by the physician and patient (mean $28+/-4$ months of treatment). In those who discontinued treatment due to lack of efficacy or worsening disease, nine out of these 12 patients had a progressive component to their disease at the time of discontinuation (75\%).

The death occurred in a patient who was 50-years-old after 21 months and 16 infusions of natalizumab treatment. The cause of death was heart failure. There was no autopsy. This patient had previously been treated with intramuscular interferon- $\beta-1 \mathrm{a}$ (Avonex $\left.{ }^{\circledR}\right)$ for one year. Neutralizing antibodies were negative in this patient.
Non-allergic side effects included breast carcinoma in one patient, herpes zoster in one patient, elevated transaminases in one patient and an episode of syncope after 14 infusions in one patient. Breast carcinoma (biopsy-confirmed grade three duct carcinoma in-situ) occurred in a 42-year-old female after 14 months of natalizumab treatment. She had been previously treated with linolomide, intramuscular interferon- $\beta$ - $1 \mathrm{a}$ (Avonex $\left.{ }^{\circledR}\right)$, subcutaneous interferon- $\beta$ - 1 b (Betaseron $\left.{ }^{\circledR}\right)$ and glatiramer acetate (Copaxone $\AA$ ). This patient is currently under treatment and the outcome is not yet known. The herpes zoster infection occurred after 34 months of natalizumab and elevated transaminases occurred after six months of treatment; both resolved with discontinuation of treatment. There were no cases of progressive multifocal leukoencephalopathy.

One patient stopped natalizumab as she desired to become pregnant. Data regarding outcome of pregnancy is not yet available.

\section{Reasons for not starting natalizumab}

Thirty-seven patients who were prescribed natalizumab did not begin treatment. Reasons for this include fear of side effects $(38 \%)$, lack of funding (24\%), chose a first-line DMT instead (27\%), contraindication to natalizumab (5\%) and distance to nearest infusion clinic (3\%) (data missing from one patient). Contraindications to natalizumab included latent tuberculosis in one patient and past history of malignant melanoma and Hepatitis $\mathrm{C}$ in another patient.

\section{Primary outcomes in those who discontinued natalizumab}

The ARR at baseline in those 43 patients who discontinued natalizumab after at least one infusion was 1.3. During natalizumab treatment, this ARR decreased to 0.3 (missing data from eight patients). After discontinuing natalizumab, the ARR increased to 0.6 (missing data from seven). Of the 36 patients with data available, $15(42 \%)$ experienced at least one relapse after discontinuing natalizumab.

In these patients, the baseline EDSS was 3.4. At 12 months of natalizumab treatment, the EDSS was 3.4 (missing data from 19), while at 24 months of natalizumab treatment, the EDSS was 2.4 (missing data from 32). After discontinuing natalizumab, the mean EDSS score in this group was 4.1 (missing data from eight). The EDSS worsened by one or more point from baseline EDSS in 15 of $35(43 \%)$ patients after discontinuing. The EDSS was conducted at a mean duration of ten months since discontinuing natalizumab.

\section{DISCUSSION}

Our findings support the conclusions of the AFFIRM ${ }^{8}$ and SENTINEL ${ }^{9}$ studies, as well as several observational studies, ${ }^{11-}$ ${ }^{17}$ regarding the safety and effectiveness of natalizumab for MS. This suggests that the findings of these randomized controlled trials generalize to clinical practice. Patients treated with natalizumab in clinical practice in Toronto benefited from treatment, with a reduction in relapse rate, stabilization in EDSS in RRMS and improvement in quality of life and energy in some patients. These findings support the hypothesis that patients with MS benefit from natalizumab, an $\alpha_{4}$ integrin antagonist, which binds to $\alpha_{4}$ integrin on leukocytes, inhibiting their migration into the central nervous system..$^{5-7}$ 
The current study included a more diverse patient population than that included in the pivotal trials. In this study, most patients were treated with a number of other disease modifying therapies prior to natalizumab and some patients with secondary progression were treated with natalizumab. Additionally, in clinical practice, a number of patients who consider natalizumab do not go on treatment for a variety of reasons and there seems to be a higher rate of discontinuation than in trials. Given the multicultural nature of Toronto, there is a broad range of ethnicities represented in our population. However, ethnicity was not reported in the majority of charts and there was likely a reporting bias, with increased reporting of ethnicity in those patients whose ethnicity varied from that typical of MS patients.

Our primary outcome findings are similar to treatment effects in the pivotal randomized controlled trials, which is likely due to the robustness of the treatment effects. We found reduction of annualized relapse rate with natalizumab treatment compared to baseline ARR in the one year prior to treatment. In addition to the effectiveness of natalizumab, regression to the mean could contribute to this observed reduction in ARR. However, there was a similar reduction in ARR when compared to the baseline ARR in the two and three years prior to treatment. This is important since disease activity is likely to be highest in the one year prior to beginning natalizumab treatment. Additionally, a large proportion of patients were also relapse-free while on natalizumab, which supports its effectiveness. We also found stabilization of disability while on natalizumab in those with RRMS, with $86 \%$ free of progression over 12 months of treatment. Our findings in RRMS patients are not only similar to the pivotal trials, ${ }^{8,9}$ but are also similar to those of observational studies conducted in several European countries. ${ }^{11-17}$ We extend these observational findings and provide evidence in support of the effectiveness of natalizumab in MS patients in Canada.

Additionally, unlike prior studies, our observational study includes some patients with secondary progressive disease who were treated with natalizumab. Most of these patients still experienced rare relapses. We found that in those with SPMS with relapses, natalizumab was still effective in reducing ARR. However, EDSS continued to worsen despite treatment with natalizumab in SPMS and fewer patients had stabilization of EDSS compared to those with RRMS. This supports the probable overall ineffectiveness of natalizumab as a treatment for secondary progressive MS. Thus, it is important to begin treatment with natalizumab while patients are still in the relapsing phase of the disease.

A secondary outcome involved qualitative analysis of responses to an open-ended question regarding the subjective impact of natalizumab. We found that a number of patients noted improvements in quality of life, energy and cognitive function. Additionally, a number of patients noted a "wearing off" of these subjective effects. There are several limitations in interpreting the findings from this qualitative analysis. The researcher interpreted the responses to an open-ended question and there was a great deal of missing data. However, it is interesting and important to note that some patients experience subjective improvements in quality of life, energy and cognitive function with natalizumab. The impact on these areas in patients treated with natalizumab in clinical practice should be further explored with valid and reliable questionnaires.
The discontinuation rate (39\%) in this study was much higher than that in $\mathrm{AFFIRM}^{8}$ (12\% either withdrew from the study or discontinued treatment). However, this higher rate of discontinuation is expected in clinical practice, as there are additional reasons for discontinuing that are not present in trials. In trials, discontinuations were due to adverse events including allergic reactions and patient choice. However, in clinical practice additional reasons for discontinuation included termination of funding and reevaluation of the risks and benefits of treatment after 24 months when the risk of progressive multifocal leukoencephalopathy may be increased. Additionally, in our patient population, patients discontinued due to lack of efficacy or worsening of disease activity, which was primarily in those with secondary progression, a group not included in the pivotal trials. Our discontinuation rate was also higher than in other post-marketing observational studies, as inclusion criteria in these other studies often requires 12 months of natalizumab treatment, thus excluding early discontinuations.

The hypersensitivity rate was $10 \%$ in this study, while it was $4 \%$ in AFFIRM ${ }^{8}$ However, due to the small sample size of our study, this rate is within the range of the confidence interval of the pivotal trials. There were positive neutralizing antibodies in $1.8 \%$ in our study, all in patients who experienced hypersensitivity reactions. This is lower than in $\operatorname{AFFIRM}^{8}(9 \%)$; however, patients were only routinely tested for neutralizing antibodies at six months of treatment or after a hypersensitivity reaction in clinical practice, while patients were tested every 12 weeks in AFFIRM. ${ }^{8}$ Thus, there was a higher rate of detection in the trial.

Serious adverse events in the current study were similar to those in AFFIRM, ${ }^{8}$ in which there were also cases of breast carcinoma, infections, abnormal liver enzymes and infusion reactions. There have also been post-marketing reports of natalizumab-induced liver injury. ${ }^{18}$ However, there were no reports of cardiac adverse events in AFFIRM or SENTINEL.

In our group of 43 patients who discontinued treatment with natalizumab, we found that disease activity returned in many following discontinuation of treatment. This group of patients was quite heterogeneous, with differing duration of natalizumab treatment, reason for discontinuing and type of MS. However, we found that after an average of ten months since discontinuing treatment, $41 \%$ experienced at least one relapse, and EDSS score had worsened from baseline in $43 \%$. Similar return in disease activity following discontinuation of natalizumab has been previously reported with return in both radiologic ${ }^{19}$ and clinical disease activity. ${ }^{20-21}$

There are several limitations to this study given its observational design. Firstly, the nonrandomized nature of the study potentially introduces confounding factors and the potential for selection bias. However, all patients referred for natalizumab at the SMH MS Clinic between 2007 and August 2009 with charts available were included to prevent selection bias. Despite this, only patients who were prescribed natalizumab in clinical practice were included, which introduces selection bias. Secondly, the lack of a control group may introduce bias as we compared ARR and EDSS during natalizumab treatment to baseline scores rather than to an independent control group. Thirdly, this study is retrospective and relied on information already available in patient charts. 
Only neurologist-determined relapses were included in the calculation of ARR and minor relapses may have been missed. The EDSS scores were determined from the documented neurologic examination, ambulation distance and ambulatory assistance in cases where the EDSS score was not explicitly documented. However, this has been done previously and most EDSS scores were performed at visits several months following the most recent relapse. Fourthly, the study was unblinded and used an open-ended question that was interpreted by the researcher to assess subjective effects of natalizumab including quality of life and energy. This can introduce bias and these areas should be further assessed with valid and reliable tools. Finally, only patients who received at least 12 months of natalizumab treatment were included in the analyses. This excludes patients who discontinued prior to 12 months of therapy (intolerant, failed treatment or dropped out) or who had not yet received 12 months of therapy, which may increase perceived treatment effects. However, our findings are important to consider in conjunction with findings from randomized controlled trials. A benefit of the observational design is that inclusion criteria were not as stringent as in randomized controlled trials, allowing greater generalizability of findings to the use of natalizumab in clinical practice.

Despite the major flaws of our observational design, our primary outcome findings were similar to results of pivotal randomized controlled and post-marketing studies of natalizumab, which suggest that patients with MS have a favourable response to treatment. Our study demonstrates a very robust treatment effect, which makes it credible even despite the flawed design. Despite limitations of our study, our findings replicate those of well-controlled studies and reproduce findings already demonstrated scientifically through randomized controlled trials. We found benefits to the disease, including reduction in relapse rate, stabilization in disability, and improved quality of life in some patients. However, compared to pivotal trials, our real world design showed higher rates of discontinuation and hypersensitivity reactions. In conjunction with pivotal trials, our findings suggest that natalizumab is relatively safe and well-tolerated by MS patients in Toronto with benefits to disease activity in RRMS.

\section{FinANCIAL SUPPORT}

This project was funded through the University of Toronto's Comprehensive Research Experience for Medical Students (CREMS) program. This project was not funded by the pharmaceutical industry.

\section{Disclosure}

Dr. Paul O'Connor has received honoraria from Biogen Idec, Sanofi Aventis, Novartis, EMD Serono, Bayer, Teva Neuroscience and Genentech. He has also received research support for clinical trials from Biogen Idec, BioMS, Sanofi Aventis, Novartis, Bayer and Genentech.

\section{REFERENCES}

1. The IFNB Multiple Sclerosis Study Group. Interferon beta- $1 \mathrm{~b}$ is effective in relapsing-remitting multiple sclerosis. I. Clinical results of a multicenter, randomized, double-blind, placebocontrolled trial. Neurology. 1993;43(4):655-61.
2. Johnson KP, Brooks BR, Cohen JA, et al. Copolymer 1 reduces relapse rate and improves disability in relapsing-remitting multiple sclerosis: results of a phase III multicenter, doubleblind, placebo-controlled trial. Neurology. 1995;45(7):1268-76.

3. Jacobs LD, Cookfair DL, Rudick RA, et al. Intramuscular interferon beta-1a for disease progression in relapsing multiple sclerosis. Ann Neurol. 1996;39(3):285-94.

4. Ebers GC, Hommes O, Hughes RAC, et al. Randomised doubleblind placebo-controlled study of interferon beta-1a in relapsing/remitting multiple sclerosis. Lancet. 1998;352(9139): 1498-504.

5. Yednock TA, Cannon C, Fritz LC, Sanchez-Madrid F, Steinman L, Karin N. Prevention of experimental autoimmune encephalomyelitis by antibodies against $\alpha 4 \beta 1$ integrin. Nature. 1992; 356(6364):63-6.

6. Baron JL, Madri JA, Ruddle NH, Hashim G, Janeway CA Jr. Surface expression of $\alpha 4$ integrin by CD4 T cells is required for their entry into brain parenchyma. J Exp Med. 1993;177:57-68.

7. Lobb RR, Mehmler ME. The pathophysiologic role of alpha 4 integrins in vivo. J Clin Invest. 1994;94(5):1722-8.

8. Polman $\mathrm{CH}, \mathrm{O}^{\prime}$ Connor PW, Havrdova E, et al. A randomized, placebo-controlled trial of natalizumab for relapsing multiple sclerosis. N Engl J Med. 2006;354:899-910.

9. Rudick RA, Stuart WH, Calabresi PA, et al. Natalizumab plus interferon beta-1 a for relapsing multiple sclerosis. N Engl J Med. 2006;354:911-23.

10. Rudick RA, Miller D, Hass S, et al. Health-related quality of life in multiple sclerosis: effects of natalizumab. Ann Neurol. 2007;62 (4):335-46.

11. Putzki N, Yaldizli O, Maurer M, et al. Efficacy of natalizumab in second line therapy of relapsing-remitting multiple sclerosis: results from a multi-center study in German speaking countries. Eur J Neurol. 2010;17(1):31-7.

12. Putzki N, Kollia K, Woods S, Egwe E, Diener HC, Limmroth V. Natalizumab is effective as second line therapy in the treatment of relapsing remitting multiple sclerosis. Eur J Neurol. 2009;16 (3):424-6.

13. Oturai AB, Koch-Henriksen N, Petersen T, Jensen PEH, Sellebjerg F, Sorensen PS. Efficacy of natalizumab in multiple sclerosis patients with high disease activity: a Danish nationwide study. Eur J Neurol. 2009;16:420-3.

14. Outteryck O, Ongagna JC, Zephir H, et al. Demographic and clinic characteristics of French patients treated with natalizumab in clinical practice. J Neurol. 2010;257:207-11.

15. Sangalli F, Moiola L, Bucello S, et al. Efficacy and tolerability of natalizumab in relapsing- remitting multiple sclerosis patients: a post-marketing observational study. Neurol Sci. 2010. Epub 2010 June 11.

16. Belachew S, Phan-Ba R, Bartholome E, et al. Natalizumab induced a rapid improvement of disability status and ambulation after failure of previous therapy in relapsing-remitting multiple sclerosis. Eur J Neurol. 2010. Epub 2010 June 16.

17. Fernandez V, Alvarenga MP, Guerrero M, et al. The efficacy of natalizumab in patients with multiple sclerosis according to level of disability: results of an observational study. Mult Scler. 2010. Epub 2010 Nov 18.

18. Bezabeth S, Flowers CM, Kortepeter C, Avigan M. Clinically significant liver injury in patients treated with natalizumab. Aliment Pharmacol Ther. 2010;31(9):1028-35.

19. Vellinga MM, Castelijns JA, Barkhof F, Uitdehaag BMJ, Polman $\mathrm{CH}$. Postwithdrawal rebound increase in T2 lesional activity in natalizumab-treated MS patients. Neurology. 2008;70(13): 1150-1.

20. Miravalle A, Jensen R, Kinkel P. Immune reconstitution inflammatory syndrome in patients with multiple sclerosis following cessation of natalizumab therapy. Arch Neurol. 2010. Epub 2010 Oct 11.

21. Borriello G, Prosperini L, Marinelli F, Fubelli F, Pozzilli C. Observations during an elective interruption of natalizumab treatment: a post-marketing study. Mult Scler. 2010. Epub 2010 Dec 9. 